\title{
The effect of Lactobacillus rhamnosus hsryfm 1301 on the intestinal microbiota of a hyperlipidemic rat model
}

Dawei Chen ${ }^{1,2}$, Zhenquan Yang ${ }^{1,2}$, Xia Chen ${ }^{1,2}$, Yujun Huang ${ }^{1,2}$, Boxing Yin 1,2, Feixiang Guo ${ }^{1,2}$, Haiqing Zhao ${ }^{1,2}$, Tangyan Zhao ${ }^{1,2}$, Henxian $\mathrm{Qu}^{1,2}$, Jiadi Huang ${ }^{3}$, Yun $\mathrm{Wu}^{3}$ and Ruixia Gu $\mathrm{G}^{1,2^{*}}$

\begin{abstract}
Background: Growing evidence indicates that intestinal microbiota regulate our metabolism. Probiotics confer health benefits that may depend on their ability to affect the gut microbiota. The objective of this study was to examine the effect of supplementation with the probiotic strain, Lactobacillus rhamnosus hsryfm 1301, on the gut microbiota in a hyperlipidemic rat model, and to explore the associations between the gut microbiota and the serum lipids.
\end{abstract}

Methods: The hyperlipidemic rat model was established by feeding rats a high-fat diet for $28 \mathrm{~d}$. The rats' gut microbiota were analyzed using high-throughput sequencing before and after L. rhamnosus hsryfm 1301 supplementation or its fermented milk for $28 \mathrm{~d}$. The serum lipids level was also tested.

Results: The rats' primary gut microbiota were composed of Bacteroidetes, Firmicutes, Proteobacteria, Spirochaetes and Verrucomicrobia. The abundance and diversity of the gut microbiota generally decreased after feeding with a high-fat diet, with a significant decrease in the relative abundance of Bacteroidetes, but with an increase in that of Firmicutes $(P<0.05)$. Administration of $L$. rhamnosus hsryfm 1301 or its fermented milk for $28 \mathrm{~d}$, could recover the Bacteroidetes and Verrucomicrobia abundance and could decrease the Firmicutes abundance, which was associated with a significant reduction in the serum lipids' level in the hyperlipidemic rats with high-fat diet induced. The abundance of 22 genera of gut bacteria was changed significantly after probiotic intervention for $28 d(P<0.05)$. A positive correlation was observed between Ruminococcus spp. and serum triglycerides, Dorea spp. and serum cholesterol (TC) and low-density lipoprotein (LDL-C), and Enterococcus spp. and high-density lipoprotein. The Butyrivibrio spp. negatively correlated with TC and LDL-C.

Conclusions: Our results suggest that the lipid metabolism of hyperlipidemic rats was improved by regulating the gut microbiota with supplementation of L.rhamnosus hsryfm 1301 or its fermented milk for $28 \mathrm{~d}$.

\section{Background}

The total number of microbes in the adult gut is $10^{14}$, which is 10 -fold higher than the number of human body cells [1]. It has been proposed that the capacity of the microbiome largely exceeds the human genome with more than three million genes [2], which are rich in carbohydrates, amino acids, vitamins, and other genes

\footnotetext{
* Correspondence: guruixia1963@163.com

${ }^{1}$ College of Food Science and Technology, Yangzhou University, Yangzhou 225127, Jiangsu Province, China

${ }^{2}$ Key Lab of Dairy Biotechnology and Safety Control, Yangzhou 225127,

Jiangsu Province, China

Full list of author information is available at the end of the article
}

involved in nutrient metabolism, most of which are absent in humans [3]. The intestinal microbes affect our lives not only through food metabolism and exclusion of pathogens, but also by modulating the mucosal immune response [4]. Hence, they have a remarkable potential to influence the physiological functions and health of the host $[5,6]$.

Probiotics are defined as live micro-organisms that confer health benefits to the gut microbiota of the host when present in adequate amounts. The function of the intestinal microbiota is not understood, yet. Nonetheless, advances in culture-independent molecular techniques have provided insight into the composition of the intestinal microbiota before and after probiotic intake [7]. 
Elevated serum lipids' level is widely recognized as a primary risk factor for the development of atherosclerosis, coronary heart disease and other cardiovascular diseases [8]. Currently, drug therapy is the principal treatment, but with high relative costs and side effects, it is not considered to be an optimal long-term treatment. Recent studies have demonstrated that Lactobacilli or Bifidobacteria could exhibit hypolipidemic effects in animal models $[9,10]$ and in humans $[11,12]$. Furthermore, the intestinal microbiota could improve the host's lipid metabolism via microbial activities [13]. The widely accepted mechanism is that microbiota activities promote bile acid biotransformation in vivo to regulate fat digestion, and affect lipid metabolism to decrease serum lipids [14].

In the present study, L. rhamnosus hsryfm 1301, which is a strong hypolipidemic agent in vitro, was isolated from the gut of subjects from Bama, Guangxi Province, China, who are known for increased longevity. As dairy products may be suitable vehicles for the delivery of probiotics and may enhance the effect of products on the host's health, L. rhamnosus hsryfm

1301-containing skim milk suspension and its fermented milk were used to investigate the effect on serum lipids and on the composition of the intestinal microbiota in hyperlipidemic rats, and to explore the possible mechanism of hypolipidemic of lactic acid bacteria in vivo.

\section{Methods}

\section{Bacteria and culture}

L. rhamnosus strain hsryfm 1301 was obtained from the Key Lab of Dairy Biotechnology and Safety Control, Yangzhou University, which was isolated from the gut of subjects from Bama longevity, Guangxi Province, China, in 2013. The bacteria were grown in MRS medium at $37^{\circ} \mathrm{C}$ in an anaerobic jar (Ruskinn Technologies, Ltd., South Wales, UK) for $24 \mathrm{~h}$.

Cells were collected by centrifugation at $4,000 \times g$ for $10 \mathrm{~min}$ at $4^{\circ} \mathrm{C}$. The L. rhamnosus hsryfm 1301-containing skim milk suspension was prepared by suspending the cells in $10 \%(\mathrm{w} / \mathrm{v})$ sterile skim milk to achieve a viable count of $10^{9} \mathrm{CFU} / \mathrm{mL}$, which was then stored at $4^{\circ} \mathrm{C}$. Tenfold serial dilutions of the suspension were performed, and $100 \mu \mathrm{L}$ were plated on MRS agar $(\mathrm{pH} 6.8 \pm 0.2)$ in triplicate. Aerobic plates were placed in an anaerobic jar (Ruskinn Technologies, Ltd.) at $37^{\circ} \mathrm{C}$ for $48 \mathrm{~h}$. The colonies counted after incubation represented the numbers of L. rhamnosus hsryfm 1301.

\section{Preparation of fermented milk}

L. rhamnosus hsryfm 1301 was added to $10 \%$ heated skim milk to a final concentration of $3 \%(\mathrm{v} / \mathrm{v})$ inoculum, and fermented at $42^{\circ} \mathrm{C}$ until the milk curdled. Fermentation was stopped when the bacterial viable count was
$10^{9} \mathrm{CFU} / \mathrm{mL}$ by bacterial counting as above. The fermented milk was stored at $4^{\circ} \mathrm{C}$.

\section{Animal trial \\ Animal groups and diets}

Thirty-eight male Sprague-Dawley (SD) rats, aged 5 weeks and weighing $140 \pm 4.5 \mathrm{~g}$ were purchased from Comparing Medical Center of Yang Zhou University (Jiangsu, China). The rats were exposed to a $12 \mathrm{~h}$ light/ dark cycle, and maintained at a constant temperature of $23 \pm 1^{\circ} \mathrm{C}$ and humidity of $50 \pm 5 \%$. The care and use of rats was according to our institutional and national guidelines, and all experiments were approved by the Ethics Committee of the Yang Zhou University.

The rats were subjected to a 1-week adaptation period on a normal diet containing $20 \%(\mathrm{w} / \mathrm{w})$ flour, $10 \%$ rice flour, $20 \%$ corn, $26 \%$ drum head, $20 \%$ bean, $2 \%$ fish powder and $2 \%$ bone powder (XieTong, Organism Inc., Jiangsu, China), The rats were randomly assigned to four groups, control and model group (11 rats each), and two treatment groups (eight rats each). The initial average body weight was similar among the four groups. The four groups were given the following diets: (1) control group, normal diet; (2) model group, high-fat diet; (3) hsryfm 1301 group, high-fat diet $+L$. rhamnosus hsryfm 1301-containing skim milk suspension; (4) hsryfm 1301-f group, high-fat diet + L. rhamnosus hsryfm 1301-containing skim fermented milk. The high-fat diet contained $10 \%$ (w/w) lard oil, $1 \%$ cholesterol, $0.2 \%$ sodium cholate and 78.8\% normal diet (XieTong, Organism Inc.). The rats had free access to water and their specific diet. The hyperlipidemic rat model was established by providing a high-fat diet for $28 \mathrm{~d}$. The hsryfm 1301 group and hsryfm 1301-f group received daily $2 \mathrm{~mL}\left(10^{9} \mathrm{CFU} / \mathrm{mL}\right)$ of $L$. rhamnosus hsryfm 1301-containing skim milk suspension and L. rhamnosus hsryfm 1301-containing skim fermented milk, respectively, which was administered intragastrically for $28 \mathrm{~d}$, after the hyperlipidemic rat model was established The control and model groups received an equivalent volume of saline. Their body weight and food consumption were measured weekly and daily, respectively.

\section{Sample collection}

Three fresh fecal samples of $1.00 \mathrm{~g}$ (wet weight) were collected from each group on day 1,28 , and 56 before feeding. Samples were suspended in $15.0 \mathrm{~mL}$ of $0.10 \mathrm{~mol} / \mathrm{L}$ phosphate buffer ( $\mathrm{pH}$ 7.4) by vortexing for $5 \mathrm{~min}$, followed by addition of $10.0 \mathrm{~mL}$ of phosphate buffer and vortexing for $5 \mathrm{~min}$. This procedure was repeated, and then samples were centrifugated at $200 \times g$ for $5 \mathrm{~min}$ to collect the supernatant (bacteria). The fecal samples were dealt with twice as above to collect the supernatant. The supernatant was centrifuged at $9000 \times g$ for $5 \mathrm{~min}$, and then the pellet was suspended in $30.0 \mathrm{~mL}$ of phosphate buffer by 
vortexing for 5 min followed by centrifugation as above. The sediment was collected and suspended in $10 \mathrm{~mL}$ of $0.10 \mathrm{~mol} / \mathrm{L}$ phosphate buffer $(\mathrm{pH} 7.4)$ by vortexing for $5 \mathrm{~min}$, aliquoted into five tubes, and kept at $-70^{\circ} \mathrm{C}$ for DNA extraction.

Three rats, which were fasted for $12 \mathrm{~h}$ and euthanized, were selected randomly from the control and model group at day 28 . Blood samples $(4 \mathrm{~mL})$ were collected into nonheparinized vacuum collection tubes from the celiac vein. Tubes were initially held stationary at $0^{\circ} \mathrm{C}$ for $30 \mathrm{~min}$, and then the serum was separated from the blood by centrifugation at $2,000 \times \mathrm{g}$ for $10 \mathrm{~min}$ at $4^{\circ} \mathrm{C}$, and was used to analyze the lipids' level.

\section{Serum lipids analysis}

Triglycerides (TG), total cholesterol (TC), high density lipoprotein cholesterol (HDL-C) and low density lipoprotein cholesterol (LDL-C) in the serum were analyzed by commercial kits (Maker, Biotechnology Inc., Sichuan, China) and the chemical analyzer 7020 (Hitachi, Tokyo, Japan). After administering the treatment intragastrically for $28 \mathrm{~d}$, all rats were weighed and the TC, TG, HDL-C and LDL-C were measured by the above mentioned methods.

\section{Gut microbiota analysis}

Microbial DNA from the fecal samples was extracted using QIAamp DNA stool mini kit (Qiagen Inc., Hilden, Germany) according to the manufacturer's instructions. The V3 hypervariable region of the $16 \mathrm{~S}$ rDNA was PCR amplified from the microbial genomic DNA using universal primer (forward primers: 5'-ACTCCTACGGGAGGCAG CAG-3', reverse primers:

5' - TTACCGCGGCTGCTGGCAC-3'). The PCR condition were $94^{\circ} \mathrm{C}$ for 4 min, followed by 21 cycles of $94^{\circ} \mathrm{C}$ for $30 \mathrm{~s}, 58^{\circ} \mathrm{C}$ for $30 \mathrm{~s}$ (annealing) and $72^{\circ} \mathrm{C}$ for $30 \mathrm{~s}$ (extension), and then $72^{\circ} \mathrm{C}$ for $5 \mathrm{~min}$.

The PCR products were excised from a 1.5\% agarose gel and purified by AxyPrep Gel Extraction Kit (Axygen, Scientific Inc., Union City, CA, USA). They were then quantified by PicoGreen dsDNA Assay Kit (Life Technologies Inc., Grand Island, NY, USA) and BioTek Microplate Reader (BioTek Inc., Winooski, VT, USA). The Barcoded V3 amplicon was sequenced using the pair-end method by Illumina Miseq at Personal Biotechnology Co., Ltd (Shanghai, China). Sequences reads with an average quality score lower than 25, ambiguous bases, homopolymer $>7$ bases, containing primer mismatches, or reads length shorter than 100 bp were removed. For V3 pairend read, only sequences that overlapped more than $10 \mathrm{bp}$ and without any mismatches were assembled [15]. Reads that could not be assembled were removed. Barcode and sequencing primers were trimmed from assembled sequences.

\section{Statistical analysis}

The SPSS software (IBM Corp, Armonk, NY, USA) was used to analyze the serum lipids data and the association between serum lipids and intestinal microbiota.

Sequences were clustered and assigned to operational taxonomic units (OTUs) using the Quantitative Insights into Microbial Ecology (QIIME) implementation of cdhit according to a threshold of $97 \%$ pairwise identity. The OTU of every sample and the number of sequences of every OTU were counted after the OTU output. The taxonomy information of every OTU was obtained by searching the most similar species. The rarefaction curve was generated by OTUs at the 97\% similarity cut-off level. Rarefaction analysis was performed in Analytic Rarefaction v.1.3 (Hunt Mountain Software, Athens, GA, USA). The Chao1 and ACE abundance indexes, and the Simpson and Shannon diversity indexes were calculated using Mothur software (http://www.mothur.org/) to analyze Alpha diversity. The SPSS (IBM Corp), Fast UniFrac (http://bmf2.colorado.edu/fastunifrac/) and QIIME (http://qiime.sourceforge.net/) software were used to analyze sequence data, bacterial community distribution and the principal component.

\section{Results}

Effects of L. rhamnosus hsryfm 1301 and its fermented milk on physical indexes of a hyperlipidemic rat model

The rats in the control and model groups were fed with normal diet or high-fat diet for $28 \mathrm{~d}$, respectively. The serum lipids' levels are summarized in Figure 1. The TC and TG levels in the model group were significantly higher than those in the control group $(P<0.05$; Figure 1$)$, indicating that the hyperlipidemia rat model was successfully established. The HDL-C and LDL-C levels in the control group were lower than those in the model group, but not significantly $(P>0.05$; Figure 1$)$.

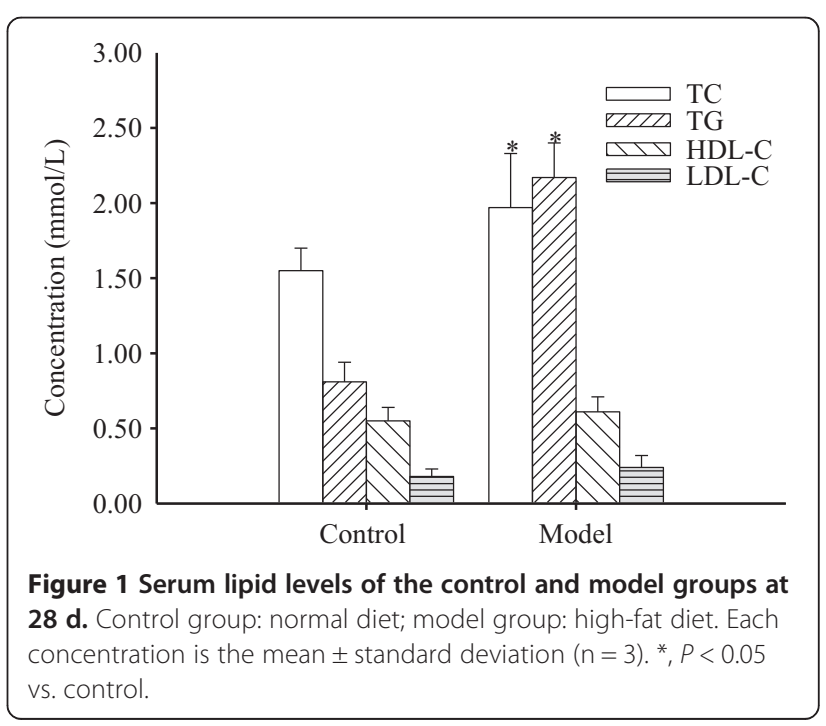


The rats' body weight increased during the study period. At the end of the experiment, the gained body weight and the average food consumption of the model group were significantly higher than those of the other groups $(P<0.05$; Figure $2 \mathrm{~A}$ and $\mathrm{B})$. The efficiency of diet utilization of the hsryfm 1301-f group was significantly higher than that of the control and model groups $(P<0.05$; Figure $2 \mathrm{C}$ ), indicating that the L. rhamnosus hsryfm fermented milk improved the efficiency of diet utilization.

After the rats were administered intragastrically $L$. rhamnosus hsryfm 1301 or its fermented milk for $28 \mathrm{~d}$, the TC, TG and HDL-C levels in the control, hsryfm 1301 and hsryfm 1301-f groups were significantly lower than those in the model group $(P<0.05$; Figure 2D). Also, the LDL-C level in the hsryfm 1301-f group was significantly lower than that in the model group $(P<0.05$; Figure 2D). The results indicate that L.rhamnosus hsryfm 1301 and its fermented milk had obvious effects on rat serum lipids.

\section{Effects of L. rhamnosus hsryfm 1301 and its fermented milk on rats intestinal microbiota Sequencing results of fecal samples}

In total, 87,228 sequence reads were obtained and grouped into 8,244 OTUs at the $97 \%$ similarity cut-off level. Among the high quality sequences, more than $95 \%$ were longer than $146 \mathrm{bp}$, with most ranging between 146 and 177 bp. The number of OTUs of Phylum, Class, Order, Family and Genus detected by sequencing was $5,359,4,348,4,140,2,583$ and 2,583, respectively.

Rarefaction analysis showed that the OTUs of 36 samples gradually increased with the increase of the number of measured sequences. Furthermore, the curve became more gentle and the increasing trend became smaller (see Additional file 1: Figure S1), indicating that most of the bacterial sequences in the samples obtained by the Illumina Miseq Sequencing system reflected the abundance and diversity of the gut microbiota.

\section{Analysis of alpha diversity of gut microbiota}

The Chao1, ACE and Shannon indexes were significantly higher and the Simpson index was significantly lower at $1 \mathrm{~d}$ compared with after feeding high-fat diet for $28 \mathrm{~d}$ or normal diet for $56 \mathrm{~d}(P<0.05$; Table 1$)$, indicating that the abundance and diversity of the gut microbiota in rats decreased with the weight increase. However, the abundance and diversity recovered or was even higher than the initial level after rats were administered intragastrically L. rhamnosus hsryfm 1301 or its fermented milk for $28 \mathrm{~d}(P<0.05$; Table 1$)$. These results showed that
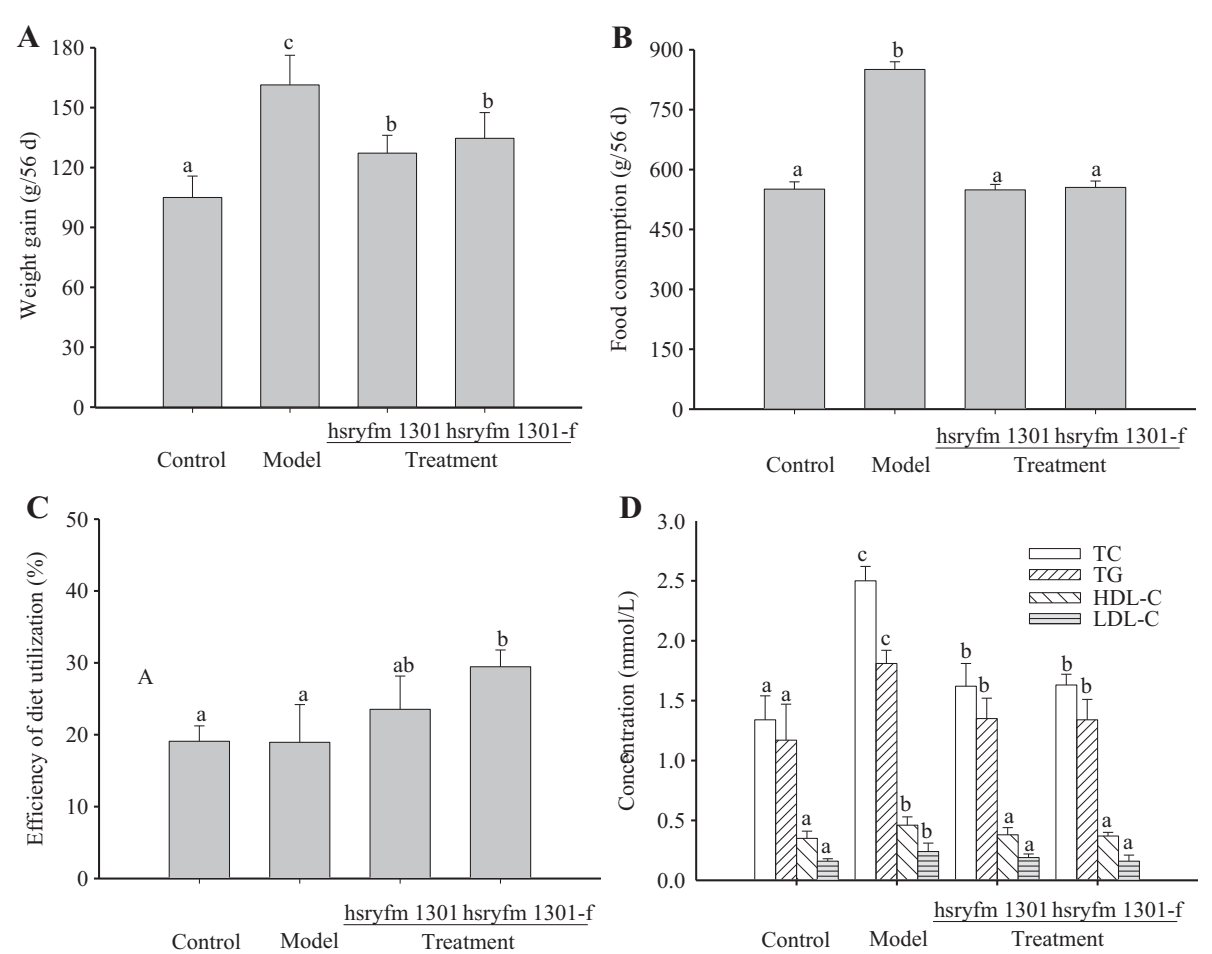

Figure 2 Physical indexes of rats. Control group: normal diet; model group: high-fat diet; hsryfm 1301 group: high-fat diet + L. rhamnosus hsryfm 1301-containing skim milk suspension; hsryfm 1301-f group: high-fat diet + L. rhamnosus hsryfm 1301-containing skim fermented milk. A: Weight gain of the rats in $56 \mathrm{~d}$; B: Food consumption of the rats in $56 \mathrm{~d}$; C: Efficiency of diet utilization of the rats; D: Serum TC, TG, HDL-C and LDL-C concentration in the rats. Efficiency of diet utilization $(\%)=($ Weight gain/Food consumption) $\times 100$. Each value is the mean \pm standard deviation $(n=8)$. A different superscript letters means significant difference in the same index $(P<0.05)$. 
Table 1 Alpha diversity of gut microbiota in rats

\begin{tabular}{|c|c|c|c|c|c|}
\hline \multirow[t]{2}{*}{ Group } & \multirow{2}{*}{$\begin{array}{l}\text { Sample } \\
\text { collection } \\
\text { time (d) }\end{array}$} & \multicolumn{2}{|c|}{ Abundance index } & \multicolumn{2}{|c|}{ Diversity index } \\
\hline & & Chao1 & ACE & Simpson & Shannon \\
\hline \multirow[t]{3}{*}{ Control } & 1 & $16332.081^{a}$ & $23580.922^{a}$ & $0.067^{b}$ & $15.876^{\mathrm{a}}$ \\
\hline & 28 & $16957.731^{\mathrm{a}}$ & $23883.323^{\mathrm{a}}$ & $0.072^{b}$ & $15.705^{\mathrm{a}}$ \\
\hline & 56 & $14196.252^{b}$ & $21689.237^{b}$ & $0.088^{\mathrm{a}}$ & $15.460^{\mathrm{b}}$ \\
\hline \multirow[t]{3}{*}{ Model } & 1 & $13796.613^{a}$ & $19983.587^{\mathrm{a}}$ & $0.063^{c}$ & $15.864^{\mathrm{a}}$ \\
\hline & 28 & $12538.624^{b}$ & $18339.896^{b}$ & $0.075^{b}$ & $15.221^{b}$ \\
\hline & 56 & $11200.145^{c}$ & $18168.955^{\mathrm{b}}$ & $0.103^{a}$ & $14.828^{c}$ \\
\hline \multirow[t]{3}{*}{ Hsryfm 1301} & 1 & $14699.544^{a}$ & $21791.684^{b}$ & $0.075^{b}$ & $15.531^{\mathrm{a}}$ \\
\hline & 28 & $12836.493^{b}$ & $19663.483^{c}$ & $0.096^{\mathrm{a}}$ & $14.763^{b}$ \\
\hline & 56 & $14947.348^{\mathrm{a}}$ & $22180.152^{\mathrm{a}}$ & $0.077^{b}$ & $15.455^{\mathrm{a}}$ \\
\hline \multirow[t]{3}{*}{ Hsryfm 1301-f } & 1 & $14725.082^{a}$ & $21799.783^{b}$ & $0.067^{b}$ & $15.740^{\mathrm{a}}$ \\
\hline & 28 & $13664.791^{b}$ & $20176.062^{c}$ & $0.073^{a}$ & $15.406^{b}$ \\
\hline & 56 & $14982.826^{a}$ & $22405.761^{\mathrm{a}}$ & $0.068^{b}$ & $15.723^{\mathrm{a}}$ \\
\hline
\end{tabular}

The Chao1, ACE, Simpson and Shannon indexes are presented for a similarity of 0.97 between the reads. Values of the same group within a column with different superscript letters mean the index differ significantly at different time points $(P<0.05, \mathrm{n}=3)$.

high-fat diet decreased the abundance and diversity of the gut microbiota in rats, while the L. rhamnosus hsryfm 1301 or its fermented milk improved them.

\section{Principal component analysis of the gut microbiota}

The sequences of 36 fecal samples from 1, 28 and $56 \mathrm{~d}$ were used for principal component analysis. The similarity between microbiota can be expressed by the BeTa diversity analysis using the unweighted UniFrac and QIIME procedure. Each point represents one sample's microbiota, and the distance between points represents the similarity between sequences of two samples' microbiota.

We found that the gut microbiota at the three tested time points were separated from each other, except for one of the $28 \mathrm{~d}$ microbiota, and that the microbiota gathered together at the same time point (see Additional file 2: Figure S2), showing that the gut microbiota in rats was significantly different at the different time points and treatments. The gut microbiota in the control group at $28 \mathrm{~d}$ was not separated from the $1 \mathrm{~d}$ microbiota, indicating that the normal diet did not affect the gut microbiota at $28 \mathrm{~d}$, and the abundance and diversity of the gut microbiota had not changed significantly (Table 1).

\section{Composition of the gut microbiota}

The gut microbiota of the rats was constituted of Bacteroidetes, Firmicutes, Proteobacteria, Spirochaetes and Verrucomicrobia at the phylum level, and the Bacteroidetes were the most abundant, followed by Firmicutes and Proteobacteria (Figure 3). After being fed a normal

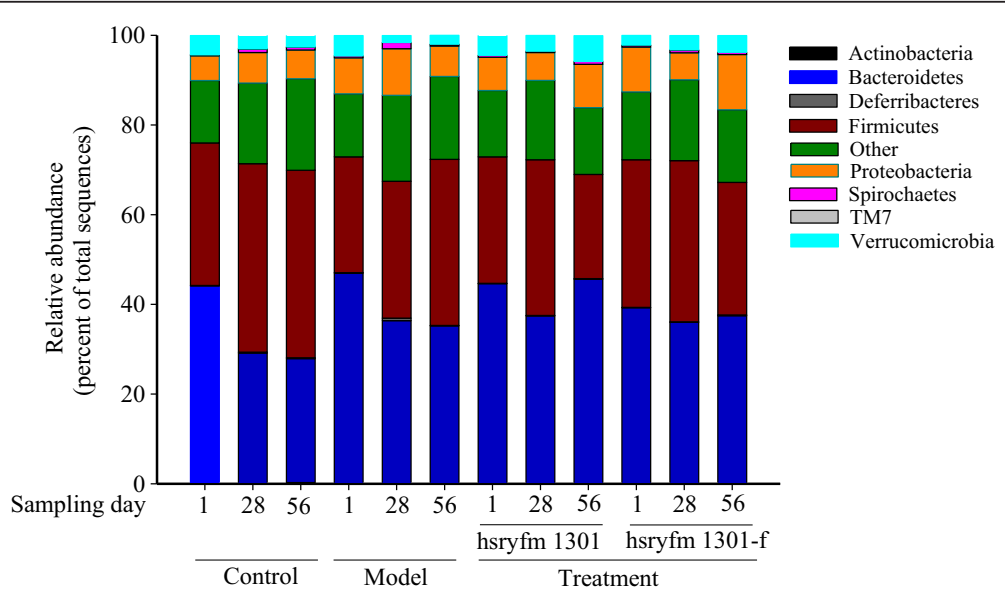

Figure 3 Relative abundance of the gut microbiota in rats at the phylum level at different time points. Control group: normal diet; model group: high-fat diet; hsryfm 1301 group: high-fat diet + L. rhamnosus hsryfm 1301-containing skim milk suspension; hsryfm 1301-f group: high-fat diet + L. rhamnosus hsryfm 1301-containing skim fermented milk. 
or high-fat diet for $56 \mathrm{~d}$, the relative abundance of Bacteroidetes and Verrucomicrobia in the rats' gut significantly decreased $(P<0.05)$ and of Firmicutes significantly increased $(P<0.05$; Table 2). Except for Bacteroidetes, opposite results were observed after treatment with $L$. rhamnosus hsryfm 1301 or its fermented milk for $28 \mathrm{~d}$ $(P<0.05$; Table 2). The relative abundance of Spirochaetes in the control, hsryfm 1301 and hsryfm 1301-f groups significantly increased, whereas it significantly decreased in the model group at $56 \mathrm{~d}$ compared with that at $1 \mathrm{~d}$ $(P<0.05$; Table 2).
Bacteroides spp., Prevotella spp., Oscillibacter spp., Helicobacter spp. and Akkermansia spp. were the primary intestinal microbiota in the rats' gut at the genus level (Table 2). The relative abundance of 22 genera of the intestinal microbiota belonging to Actinobacteria, Bacteroidetes, Deferribacteres, Firmicutes, Proteobacteria, Spirochaetes and Verrucomicrobia was significantly different after being fed a high-fat diet for $56 \mathrm{~d}$ and administered intragastrically L. rhamnosus hsryfm 1301 or its fermented milk for $28 \mathrm{~d}(P<0.05$; Table 2). The serum lipids in the control, hsryfm 1301 and hsryfm 1301-f

Table 2 Difference in abundance of intestinal microbiota in rats

\begin{tabular}{|c|c|c|c|c|c|c|c|c|}
\hline \multirow[b]{3}{*}{ Taxon } & \multicolumn{8}{|c|}{ Relative abundance (percent of total sequences) } \\
\hline & \multicolumn{4}{|c|}{$1 \mathrm{~d}$} & \multicolumn{4}{|c|}{$56 \mathrm{~d}$} \\
\hline & Control & Model & hsryfm 1301 & hsryfm 1301-f & Control & Model & hsryfm 1301 & hsryfm 1301-f \\
\hline Actinobacteria & $0.10 \pm 0.01$ & $0.17 \pm 0.02$ & $0.20 \pm 0.01$ & $0.13 \pm 0.01$ & $0.27 \pm 0.01$ & $0.17 \pm 0.02$ & $0.13 \pm 0.01$ & $0.13 \pm 0.01$ \\
\hline Collinsella & $0.00 \pm 0.00$ & $0.10 \pm 0.01$ & $0.10 \pm 0.01$ & $0.06 \pm 0.01$ & $0.17 \pm 0.02^{*}$ & $0.09 \pm 0.001$ & $0.10 \pm 0.01$ & $0.10 \pm 0.01^{* *}$ \\
\hline Bacteroidetes & $44.00 \pm 2.50$ & $46.80 \pm 1.56$ & $44.40 \pm 2.12$ & $39.10 \pm 1.20$ & $27.63 \pm 1.53^{* *}$ & $35.03 \pm 1.20^{* *}$ & $45.50 \pm 2.62$ & $40.33 \pm 1.72$ \\
\hline Bacteroides & $1.47 \pm 0.21$ & $2.43 \pm 0.12$ & $2.33 \pm 0.15$ & $1.93 \pm 0.20$ & $1.10 \pm 0.16$ & $1.40 \pm 0.11^{*}$ & $4.50 \pm 0.26^{* *}$ & $3.33 \pm 0.28^{* *}$ \\
\hline Barnesiella & $0.10 \pm 0.01$ & $0.07 \pm 0.01$ & $0.08 \pm 0.02$ & $0.08 \pm 0.01$ & $0.17 \pm 0.01$ & $0.10 \pm 0.01$ & $0.00 \pm 0.00^{*}$ & $0.00 \pm 0.00^{*}$ \\
\hline Parabacteroides & $0.30 \pm 0.02$ & $0.47 \pm 0.03$ & $0.40 \pm 0.02$ & $0.33 \pm 0.01$ & $0.20 \pm 0.01$ & $0.30 \pm 0.02^{*}$ & $0.45 \pm 0.01^{*}$ & $0.40 \pm 0.01$ \\
\hline Alistipes & $0.30 \pm 0.02$ & $0.10 \pm 0.01$ & $0.10 \pm 0.01$ & $0.13 \pm 0.01$ & $0.10 \pm 0.01^{*}$ & $0.17 \pm 0.01^{*}$ & $0.05 \pm 0.01^{*}$ & $0.13 \pm 0.01$ \\
\hline Prevotella & $20.47 \pm 1.02$ & $22.77 \pm 1.13$ & $18.93 \pm 1.08$ & $17.67 \pm 1.12$ & $16.10 \pm 2.03^{*}$ & $14.83 \pm 1.16^{* *}$ & $21.03 \pm 1.15^{*}$ & $17.57 \pm 1.09$ \\
\hline Deferribacteres & $0.10 \pm 0.01$ & $0.10 \pm 0.02$ & $0.10 \pm 0.01$ & $0.10 \pm 0.01$ & $0.20 \pm 0.01^{*}$ & $0.10 \pm 0.01$ & $0.10 \pm 0.02$ & $0.17 \pm 0.02^{*}$ \\
\hline Mucispirillum & $0.10 \pm 0.01$ & $0.10 \pm 0.02$ & $0.10 \pm 0.01$ & $0.10 \pm 0.01$ & $0.13 \pm 0.01$ & $0.10 \pm 0.01$ & $0.10 \pm 0.02$ & $0.17 \pm 0.02^{*}$ \\
\hline Firmicutes & $31.80 \pm 2.05$ & $25.83 \pm 1.53$ & $28.20 \pm 1.74$ & $32.90 \pm 1.85$ & $41.80 \pm 2.05^{*}$ & $37.03 \pm 1.64^{*}$ & $23.23 \pm 1.26^{*}$ & $27.57 \pm 1.02^{*}$ \\
\hline Lactobacillus & $0.10 \pm 0.02$ & $0.10 \pm 0.01$ & $0.10 \pm 0.01$ & $0.10 \pm 0.02$ & $0.12 \pm 0.01$ & $0.10 \pm 0.02$ & $0.17 \pm 0.01^{*}$ & $0.27 \pm 0.02^{*}$ \\
\hline Butyrivibrio & $0.13 \pm 0.01$ & $0.15 \pm 0.01$ & $0.07 \pm 0.01$ & $0.10 \pm 0.01$ & $0.15 \pm 0.01$ & $0.10 \pm 0.01$ & $0.20 \pm 0.02^{*}$ & $0.27 \pm 0.02^{* *}$ \\
\hline Dorea & $0.13 \pm 0.02$ & $0.14 \pm 0.01$ & $0.17 \pm 0.02$ & $0.17 \pm 0.02$ & $0.09 \pm 0.01$ & $0.23 \pm 0.01^{*}$ & $0.12 \pm 0.01^{*}$ & $0.10 \pm 0.01^{*}$ \\
\hline Enterococcus & $0.10 \pm 0.01$ & $0.10 \pm 0.01$ & $0.10 \pm 0.01$ & $0.10 \pm 0.01$ & $0.15 \pm 0.01^{*}$ & $0.37 \pm 0.01^{* *}$ & $0.05 \pm 0.01^{*}$ & $0.06 \pm 0.01^{*}$ \\
\hline Oscillibacter & $1.63 \pm 0.14$ & $1.60 \pm 0.11$ & $2.07 \pm 0.16$ & $2.60 \pm 0.21$ & $2.33 \pm 0.18^{*}$ & $3.37 \pm 0.31^{* *}$ & $1.43 \pm 0.13^{*}$ & $1.83 \pm 0.18^{*}$ \\
\hline Ruminococcus & $0.30 \pm 0.02$ & $0.10 \pm 0.01$ & $0.20 \pm 0.01$ & $0.20 \pm 0.01$ & $0.57 \pm 0.04^{* *}$ & $0.37 \pm 0.04^{* *}$ & $0.16 \pm 0.02^{*}$ & $0.17 \pm 0.02^{*}$ \\
\hline Allobaculum & $0.20 \pm 0.01$ & $0.27 \pm 0.02$ & $0.20 \pm 0.01$ & $0.20 \pm 0.01$ & $0.24 \pm 0.02$ & $0.10 \pm 0.01^{*}$ & $0.47 \pm 0.03^{* *}$ & $0.27 \pm 0.02$ \\
\hline Acetanaerobacterium & $0.00 \pm 0.00$ & $0.00 \pm 0.00$ & $0.07 \pm 0.01$ & $0.00 \pm 0.00$ & $0.10 \pm 0.01^{*}$ & $0.00 \pm 0.00$ & $0.10 \pm 0.01$ & $0.03 \pm 0.001^{*}$ \\
\hline Holdemania & $0.00 \pm 0.00$ & $0.07 \pm 0.01$ & $0.00 \pm 0.00$ & $0.00 \pm 0.00$ & $0.00 \pm 0.00$ & $0.00 \pm 0.00^{*}$ & $0.10 \pm 0.01^{*}$ & $0.03 \pm 0.01^{*}$ \\
\hline Proteobacteria & $5.50 \pm 1.05$ & $8.03 \pm 1.62$ & $7.43 \pm 1.16$ & $10.00 \pm 1.78$ & $6.40 \pm 0.76$ & $6.80 \pm 0.85$ & $9.67 \pm 1.13$ & $12.30 \pm 1.19$ \\
\hline Campylobacter & $0.13 \pm 0.01$ & $0.13 \pm 0.02$ & $0.10 \pm 0.01$ & $0.10 \pm 0.01$ & $0.11 \pm 0.01$ & $0.12 \pm 0.01$ & $0.00 \pm 0.00^{*}$ & $0.00 \pm 0.00^{*}$ \\
\hline Helicobacter & $0.97 \pm 0.12$ & $1.17 \pm 0.19$ & $0.77 \pm 0.05$ & $2.40 \pm 0.19$ & $1.27 \pm 0.22$ & $1.20 \pm 0.15$ & $1.13 \pm 0.21$ & $1.27 \pm 0.16^{*}$ \\
\hline Escherichia/Shigella & $0.13 \pm 0.01$ & $0.10 \pm 0.01$ & $0.07 \pm 0.01$ & $0.13 \pm 0.01$ & $0.10 \pm 0.002$ & $0.07 \pm 0.01$ & $0.00 \pm 0.00^{*}$ & $0.03 \pm 0.001^{*}$ \\
\hline Spirochaetes & $0.20 \pm 0.02$ & $0.43 \pm 0.03$ & $0.43 \pm 0.02$ & $0.33 \pm 0.02$ & $0.60 \pm 0.03^{* *}$ & $0.30 \pm 0.02^{*}$ & $0.63 \pm 0.04^{*}$ & $0.50 \pm 0.02^{*}$ \\
\hline Treponema & $0.20 \pm 0.01$ & $0.43 \pm 0.03$ & $0.43 \pm 0.02$ & $0.33 \pm 0.02$ & $0.40 \pm 0.04^{* *}$ & $0.30 \pm 0.02^{*}$ & $0.60 \pm 0.03^{*}$ & $0.50 \pm 0.04^{*}$ \\
\hline Verrucomicrobia & $4.30 \pm 0.32$ & $4.53 \pm 0.36$ & $4.50 \pm 0.38$ & $2.17 \pm 0.23$ & $2.63 \pm 0.31^{*}$ & $2.00 \pm 0.18^{* *}$ & $5.73 \pm 0.51^{*}$ & $3.73 \pm 0.32^{*}$ \\
\hline Akkermansia & $4.23 \pm 0.30$ & $4.50 \pm 0.35$ & $4.50 \pm 0.38$ & $2.13 \pm 0.21$ & $2.60 \pm 0.29^{*}$ & $2.00 \pm 0.18^{* *}$ & $5.73 \pm 0.51^{*}$ & $3.70 \pm 0.25^{*}$ \\
\hline TM7 & $0.10 \pm 0.01$ & $0.00 \pm 0.00$ & $0.00 \pm 0.00$ & $0.10 \pm 0.01$ & $0.10 \pm 0.01$ & $0.10 \pm 0.01^{*}$ & $0.00 \pm 0.00$ & $0.00 \pm 0.00^{*}$ \\
\hline Other & $13.90 \pm 1.35$ & $14.03 \pm 1.42$ & $14.77 \pm 1.51$ & $15.13 \pm 1.47$ & $20.40 \pm 2.14^{*}$ & $18.47 \pm 1.78$ & $14.90 \pm 1.65$ & $16.20 \pm 1.55$ \\
\hline
\end{tabular}

Each value is the mean \pm standard deviation $(n=3)$. Data were analyzed using one-way ANOVA. Differences between treatment periods were assessed by the Duncan test. *means values in the same row of $56 \mathrm{~d}$ are significantly different from those of $1 \mathrm{~d}(P<0.05) ;{ }^{*}, P<0.01$. 
groups were also significantly different compared with the model group at $56 \mathrm{~d}(P<0.05$; Figure 2D).

L. rhamnosus hsryfm 1301 and its fermented milk significantly decreased the relative abundance of Barnesiella spp., Dorea spp., Enterococcus spp., Oscillibacter spp., Ruminococcus spp., Campylobacter spp. and Escherichial Shigella spp. $(P<0.05)$, while they significantly increased the relative abundance of Bacteroides spp., Lactobacillus spp., Butyrivibrio spp., Holdemania spp., Treponema spp. and Akkermansia spp. $(P<0.05$; Table 2). L. rhamnosus hsryfm 1301 significantly increased the relative abundance of Parabacteroides spp., Prevotella spp., Allobaculum spp. and Psychrobacter spp. $(P<0.05)$, while it significantly decreased that of Alistipes spp. $(P<0.05$; Table 2$)$. L. rhamnosus hsryfm 1301 fermented milk significantly increased the relative abundance of Collinsella spp., Mucispirillum spp. and Acetanaerobacterium spp. $(P<0.05)$, while it significantly decreased the relative abundance of Helicobacter spp. $(P<0.05$; Table 2$)$.

\section{Discussion}

Recently, it has been reported that the host intestinal microbiota depends not only on hereditary factors, but also on environmental factors including age, diet and living environment [16]. Diet intervention is controllable, and can improve the intestinal microbiota for the longterm and induce beneficial changes [17]. Our findings provide evidence for an important modification of the intestine resulting from a probiotic treatment, and indicate its contribution to improvement of host serum lipids.

Secondary bile acid, hydrogen sulfide and other harmful products are produced in rats during lipid metabolism after a high-fat diet intervention, which harm the colorectal mucosa and damage the micro environment of the intestine that helps bacteria survive $[18,19]$. Consistently, we showed that high-fat diet decreased the abundance and diversity of the intestinal microbiota in rats, and that the abundance of Bacteroidetes and Firmicutes decreased and increased, respectively, in rats' gut. However, some polysaccharides, bile acid and steroids in the diet could be absorbed and metabolized by Bacteroidetes, which the host cannot metabolize [20], and the calories in the food could be absorbed by Firmicutes, which promotes fat storage in the host body [21]. The relative abundance of Akkermansia spp. also decreased after a high-fat diet, which is consistent with Everard et al. [22]. Akkermansia spp. can improve the gastrointestinal mucosal barrier by increasing the rat gastrointestinal mucus layer thickness, and thus prevent some harmful substances from passing through the intestine into the blood, forestall fat mass storage in vivo and ameliorate high serum lipid-related metabolic diseases caused by obesity [22].

Lactic acid bacteria can compete for intestinal nutrients, and occupy some adsorption sites and metabolites to improve the intestinal environment in rats $[23,24]$. Consistent with Xie et al. [25], the harmful bacteria Escherichia/Shigella spp. and the probiotic Lactobacillus spp. were suppressed and promoted, respectively, in the rats' gut after administering intragastrically L.rhamnosus hsryfm 1301 or its fermented milk for $28 \mathrm{~d}$. The rats' serum lipids and efficiency of diet utilization improved by L. rhamnosus hsryfm 1301 or its fermented milk, which also recovered the abundance and diversity of the intestinal microbiota, which showed increased and decreased abundance of Bacteroidetes and Firmicutes, respectively. The short chain fatty acids produced by the recovered intestinal microflora reduced the TG and TC level by inhibiting the hepatic lipogenic enzyme activity and regulating the cholesterol distribution in the blood and liver [26,27]. Accordingly, L. rhamnosus hsryfm 1301 and its fermentation products kept the balance of intestinal microbiota in rats, to alleviate the adverse effects induced by a high-fat diet.

Significant differences were observed in 22 genera of intestinal bacteria in the hsryfm 1301 and its fermented milk groups (Table 2). In fermented milk, not only the Lactobacillus itself, but also the fermentation products can have probiotic effects on the intestinal microbiota [28]. Recently, a study has shown that fermented milk could increase the number of cells with cytokines, which reduce cell death and enhance the function of the thymus to improve the mucosa and immune system of the

Table 3 Associations between serum lipids and intestinal microbiota

\begin{tabular}{cccccccc}
\hline & TC & LDL & HDL & Dorea spp. & Butyrivibrio spp. & Enterococcus spp. & Ruminococcus spp. \\
\hline TG & 0.745 & 0.65 & 0.349 & -0.305 & -0.344 & -0.422 & $0.982^{*}$ \\
TC & & 0.799 & 0.778 & $0.951^{*}$ & $-0.646^{*}$ & 0.334 & 0.06 \\
LDL & & & 0.592 & $0.988^{*}$ & $-0.747^{*}$ & 0.109 & 0.025 \\
HDL & & & & -0.122 & -0.408 & $0.953^{*}$ & 0.471 \\
Dorea spp. & & & & & -0.175 & 0.41 \\
Butyrivibrio spp. & & & & & -0.897 & 0.144 \\
Enterococcus spp. & & & & & & & 0.751 \\
\hline
\end{tabular}

The SPSS Pearson analysis was used to calculate the Pearson's correlation coefficient $(r){ }^{*}, P<0.05$. The sample sequencing of rats corresponds with the serum lipids of rats at $56 d(n=3)$. 
host, thus balancing the gut ecology [28]. On the other hand, the fermented milk could improve the expression of the microbiome, which participates in lipid and carbohydrate metabolism [29]. The TC and LDL-C levels in the hsryfm 1301-f group were lower than those in the hsryfm 1301 group, possibly because the L. rhamnosus hsryfm 1301 fermented milk could enhance the expression of bacteria that improve lipid metabolism, such as Butyrivibrio spp. (Table 2).

Analysis of the association between intestinal microbiota and serum lipids at $56 \mathrm{~d}$ showed a positive correlation between bacteria related to Ruminococcus spp. and TG, Dorea spp. and TC and LDL-C, and between Enterococcus spp. and HDL-C. The positive correlation between Ruminococcus spp. and Dorea spp., which belong to Clostridium, and serum lipids $(P<0.05$; Table 3$)$, is consistent with the findings by Lahti et al. [30], who found that Ruminococcus spp. was enriched by polyunsaturated and odd-chain fatty acids, which are not synthesized in the body, and that Ruminococcus spp. facilitates the absorption of polyunsaturated dietary lipids. The bacteria related to Butyrivibrio spp. negatively correlated with TC and LDL-C $(P<0.05$; Table 3$)$, and Butyrivibrio spp. has the c9, tll activity of linoleate isomerase [31], which could decrease the mRNA expression of SREBP-1c [32]. Sterol regulatory element-binding protein (SREBP)-1c is one of the important elements that adjusts lipid metabolism $[33,34]$, thus the increase in abundance of Butyrivibrio spp. could lower the lipid levels in rats.

\section{Conclusions}

We suggest that the intestinal microbiota and serum lipids in rats improved by feeding $L$. rhamnosus hsryfm 1301 or its fermented milk for $28 \mathrm{~d}$. The correlation between serum lipids and Ruminococcus spp., Dorea spp. and Enterococcus spp. was positive $(P<0.05)$, while the correlation between Butyrivibrio spp. and serum lipids was negative $(P<0.05)$. We believe that long-term continuous monitoring of changes in intestinal microbiota will provide further insight into the role of intestinal microbiota in human lipid metabolism.

\section{Additional files}

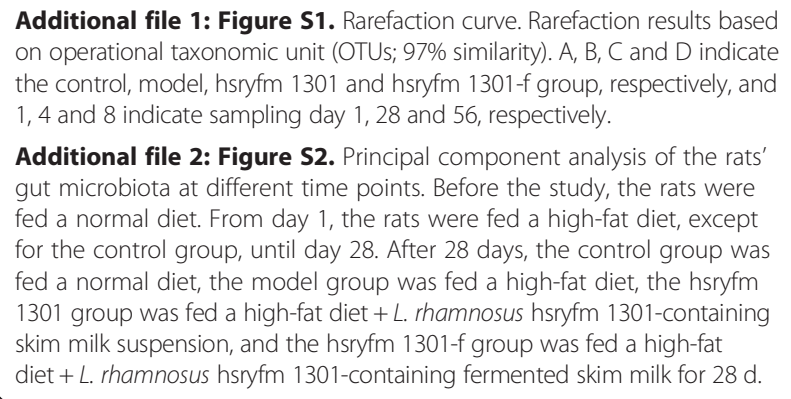

Additional file 2: Figure S2. Principal component analysis of the rats' gut microbiota at different time points. Before the study, the rats were fed a normal diet. From day 1 , the rats were fed a high-fat diet, except for the control group, until day 28. After 28 days, the control group was fed a normal diet, the model group was fed a high-fat diet, the hsryfm 1301 group was fed a high-fat diet + L. rhamnosus hsryfm 1301-containing skim milk suspension, and the hsryfm 1301-f group was fed a high-fat diet + L. rhamnosus hsryfm 1301-containing fermented skim milk for $28 \mathrm{~d}$.

\section{Abbreviations}

WHO: World Health Organization; CFU: Colony-Forming Units.

\section{Competing interests}

The authors declare that they have no competing interests.

\section{Authors' contributions}

This study was designed by CDW and GRX; CDW, CX, HYJ, YBX, GFX, ZHQ, ZTY and QHX were involved in experiment conduction and analysis; CDW, YZQ, WY and HJD performed data analysis and wrote the draft of the manuscript; GRX and YZQ provided significant academic advice and consultation. All authors read and approved the final manuscript.

\section{Acknowledgments}

This study was supported by National Key Technology Research and Development Program of the Ministry of Science and Technology of China (2013BAD18B12), the Key University Science Research Project of Jiangsu Province (12KJA550003) and National Natural Science Foundation of China (31201393).

\section{Author details}

${ }^{1}$ College of Food Science and Technology, Yangzhou University, Yangzhou 225127, Jiangsu Province, China. ${ }^{2}$ Key Lab of Dairy Biotechnology and Safety Control, Yangzhou 225127, Jiangsu Province, China. ${ }^{3}$ Royal Dairy (Guangxi) Co., Ltd., Nanning, Guangxi 530007, China.

Received: 9 June 2014 Accepted: 29 September 2014

Published: 10 October 2014

\section{References}

1. Hooper LV, Gordon J: Commensal host-bacterial relationships in the gut. Science 2001, 292(5519):1115-1118.

2. Qin J, Li R, Raes J, Arumugam M, Burgdorf KS, Manichanh C, Nielsen T, Pons $\mathrm{N}$, Levenez $\mathrm{F}$, Yamada T: A human gut microbial gene catalogue established by metagenomic sequencing. Nature 2010, 464(7285):59-65.

3. Gill SR, Pop M, DeBoy RT, Eckburg PB, Turnbaugh PJ, Samuel BS, Gordon JI, Relman DA, Fraser-Liggett CM, Nelson KE: Metagenomic analysis of the human distal gut microbiome. Science 2006, 312(5778):1355-1359.

4. Holmes E, Li JV, Athanasiou T, Ashrafian H, Nicholson JK: Understanding the role of gut microbiome-host metabolic signal disruption in health and disease. Trends Microbiol 2011, 19(7):349-359.

5. Tappenden KA, Deutsch AS: The physiological relevance of the intestinal microbiota-contributions to human health. J Am Coll Nutr 2007, 26(6):679S-683S.

6. Tremaroli $V$, Bäckhed F: Functional interactions between the gut microbiota and host metabolism. Nature 2012, 489(7415):242-249.

7. Zoetendal E, Rajilić-Stojanović M, De Vos W: High-throughput diversity and functionality analysis of the gastrointestinal tract microbiota. Gut 2008, 57(11):1605-1615.

8. WHO: Cardiovascular disease. In Fact Sheet N³17. Geneva, Switzerland: 2009. [http://www.who.int/mediacentre/factsheets/fs317/en/], (accessed on 19 May 2010).

9. Fukushima M, Nakano M: Effects of a mixture of organisms, Lactobacillus acidophilus or Streptococcus faecalis on cholesterol metabolism in rats fed on a fat-and cholesterol-enriched diet. Br J Nutr 1996, 76(06):857-867.

10. Kumar M, Rakesh S, Nagpal R, Hemalatha R, Ramakrishna A, Sudarshan V, Ramagoni R, Shujauddin M, Verma V, Kumar A: Probiotic Lactobacillus rhamnosus GG and Aloe vera gel improve lipid profiles in hypercholesterolemic rats. Nutrition 2013, 29(3):574-579.

11. Jones ML, Martoni CJ, Parent M, Prakash S: Cholesterol-lowering efficacy of a microencapsulated bile salt hydrolase-active Lactobacillus reuteri NCIMB 30242 yoghurt formulation in hypercholesterolaemic adults. Br J Nutr 2012, 107(10):1505-1513.

12. Xiao J, Kondo S, Takahashi N, Miyaji K, Oshida K, Hiramatsu A, Iwatsuki K, Kokubo S, Hosono A: Effects of Milk Products Fermented by Bifidobacterium longum on Blood Lipids in Rats and Healthy Adult Male Volunteers. J Dairy Sci 2003, 86(7):2452-2461.

13. Fava F, Lovegrove J, Gitau R, Jackson K, Tuohy K: The gut microbiota and lipid metabolism: implications for human health and coronary heart disease. Curr Med Chem 2006, 13(25):3005-3021. 
14. Ridlon JM, Kang D-J, Hylemon PB: Bile salt biotransformations by human intestinal bacteria. J Lipid Res 2006, 47(2):241-259.

15. Tian D, Ma X, Li Y, Zha L, Wu Y, Zou X, Liu S: [Research on soil bacteria under the impact of sealed $\mathrm{CO} 2$ leakage by high-throughput sequencing technology]. Huan jing ke xue= Huanjing kexue/[bian ji, Zhongguo ke xue yuan huan jing ke xue wei yuan hui" Huan jing ke xue" bian ji wei yuan hui] 2013, 34(10):4096-4104.

16. Natividad JM, Huang X, Slack E, Jury J, Sanz Y, David C, Denou E, Yang P, Murray J, McCoy KD: Host responses to intestinal microbial antigens in gluten-sensitive mice. PloS One 2009, 4(7):e6472.

17. Ley RE, Turnbaugh PJ, Klein S, Gordon Jl: Microbial ecology: human gut microbes associated with obesity. Nature 2006, 444(7122):1022-1023.

18. Attene-Ramos MS, Nava GM, Muellner MG, Wagner ED, Plewa MJ, Gaskins HR: DNA damage and toxicogenomic analyses of hydrogen sulfide in human intestinal epithelial FHs 74 Int cells. Environ Mol Mutagen 2010, 51(4):304-314

19. Resta SC: Effects of probiotics and commensals on intestinal epithelial physiology: implications for nutrient handling. J Physiol 2009, 587(17):4169-4174.

20. Xu J, Bjursell MK, Himrod J, Deng S, Carmichael LK, Chiang HC, Hooper LV, Gordon Jl: A genomic view of the human-Bacteroides thetaiotaomicron symbiosis. Science 2003, 299(5615):2074-2076.

21. Turnbaugh PJ, Ley RE, Mahowald MA, Magrini V, Mardis ER, Gordon Jl: An obesity-associated gut microbiome with increased capacity for energy harvest. Nature 2006, 444(7122):1027-1131.

22. Everard A, Belzer C, Geurts L, Ouwerkerk JP, Druart C, Bindels LB, Guiot $Y$, Derrien M, Muccioli GG, Delzenne NM: Cross-talk between Akkermansia muciniphila and intestinal epithelium controls diet-induced obesity. Proc Natl Acad Sci 2013, 110(22):9066-9071.

23. Collado M, Meriluoto J, Salminen S: Role of commercial probiotic strains against human pathogen adhesion to intestinal mucus. Lett Appl Microbiol 2007, 45(4):454-460

24. Ganz T: Antimicrobial polypeptides in host defense of the respiratory tract. J Clin Invest 2002, 109(6):693-697.

25. Xie N, Cui Y, Yin Y-N, Zhao X, Yang J-W, Wang Z-G, Fu N, Tang Y, Wang X-H, Liu X-W: Effects of two Lactobacillus strains on lipid metabolism and intestinal microflora in rats fed a high-cholesterol diet. BMC Complement Altern Med 2011, 11(1):53.

26. Larkin TA, Astheimer LB, Price WE: Dietary combination of soy with a probiotic or prebiotic food significantly reduces total and LDL cholesterol in mildly hypercholesterolaemic subjects. Eur J Clin Nutr 2009, 63(2):238-245

27. Pereira DI, McCartney AL, Gibson GR: An in vitro study of the probiotic potential of a bile-salt-hydrolyzing Lactobacillus fermentum strain, and determination of its cholesterol-lowering properties. Appl Environ Microbiol 2003, 69(8):4743-4752.

28. Núñez IN, Galdeano CM, Carmuega E, Weill R, De Moreno De LeBlanc A, Perdigón G: Effect of a probiotic fermented milk on the thymus in Balb/c mice under non-severe protein-energy malnutrition. Br J Nutr 2013, 110(03):500-508

29. McNulty NP, Yatsunenko T, Hsiao A, Faith JJ, Muegge BD, Goodman AL, Henrissat B, Oozeer R, Cools-Portier S, Gobert G: The impact of a consortium of fermented milk strains on the gut microbiome of gnotobiotic mice and monozygotic twins. Sci Transl Med 2011, 3(106):106ra. 106-106ra106.

30. Lahti L, Salonen A, Kekkonen RA, Salojärvi J, Jalanka-Tuovinen J, Palva A, Orešic M, de Vos WM: Associations between the human intestinal microbiota. Lactobacillus rhamnosus $\mathrm{GG}$ and serum lipids indicated by integrated analysis of high-throughput profiling data. PeerJ 2013, 1:e32.

31. Rainio A, Vahvaselkä M, Suomalainen T, Laakso S: Production of conjugated linoleic acid by Propionibacterium freudenreichii ssp. shermanii. Lait 2002, 82(1):91-101

32. Roche HM, Noone E, Sewter C, Mc Bennett S, Savage D, Gibney MJ, O'Rahilly S, Vidal-Puig AJ: Isomer-dependent metabolic effects of conjugated linoleic acid insights from molecular markers sterol regulatory element-binding protein-1C and LXRa. Diabetes 2002, 51(7):2037-2044.
33. Raghow R, Yellaturu C, Deng X, Park EA, Elam MB: SREBPs: the crossroads of physiological and pathological lipid homeostasis. Trends Endocrinol Metab 2008, 19(2):65-73.

34. Rubin D, Schneider-Muntau A, Klapper M, Nitz I, Helwig U, Fölsch UR, Schrezenmeir J, Döring F: Functional analysis of promoter variants in the microsomal triglyceride transfer protein (MTTP) gene. Hum Mutat 2008, 29(1):123-129.

doi:10.1186/1472-6882-14-386

Cite this article as: Chen et al:: The effect of Lactobacillus rhamnosus hsryfm 1301 on the intestinal microbiota of a hyperlipidemic rat model. BMC Complementary and Alternative Medicine 2014 14:386.

\section{Submit your next manuscript to BioMed Central and take full advantage of:}

- Convenient online submission

- Thorough peer review

- No space constraints or color figure charges

- Immediate publication on acceptance

- Inclusion in PubMed, CAS, Scopus and Google Scholar

- Research which is freely available for redistribution

Submit your manuscript at www.biomedcentral.com/submit
C Biomed Central 